\title{
Klotho suppresses RIG-I-mediated senescence-associated inammation
}

\section{Feng Liu, Su Wu, Hongwei Ren and Jun Gu}

Nat. Cell Biol. 13, 254-262 (2011); published online 20 February 2011; corrected after print 16 March 2011

In the version of this article initially published online and in print, the middle graph in Figure 3b was incorrectly labelled. Additionally two sentences on page 257 have been altered to; 'Interestingly, knockdown of RIG-I in senescent HUVECs led to extension of the lifespan of the senescent cells (Fig. 2g)...' and 'This is consistent with observations that inhibition of IL-6 and IL-8 extends the proliferative capacity of cultured cells passages ${ }^{4,5,7}$.. In the legend for figure $4 c$, wording has been changed to: '(c) Growth curves of control HUVECs and klotho-overexpressing HUVECs. Senescent HUVECs were transfected with control plasmid or klotho-expression plasmid'. On page 258, il-8 should have been IL-8. These errors have been corrected in both the HTML and PDF versions of the article. 